\title{
Olfactory Device for Large Scale Pre-screening for COVID-19
}

\author{
Prasanna Gandhi ${ }^{1} \cdot$ Ratnesh Bafna $^{1} \cdot$ Girish Arabale $^{1} \cdot$ Sunu Engineer ${ }^{1} \cdot$ Sanjay Phadke $^{1}$
}

Received: 15 April 2020 / Revised: 5 June 2020 / Accepted: 10 June 2020 / Published online: 26 June 2020

(C) Indian National Academy of Engineering 2020

\begin{abstract}
Recent pandemic caused by COVID-19 has motivated lockdown in several countries for long duration and will have a long lasting impact on the social life and economics world wide. India is no exception although showing relatively delayed increase in number of cases. Multilayered diagnostics for mass scaled screening is inevitable during and especially after the peak is passed and life is moving towards normalcy. First level of screening currently used in India is mainly based on temperature and has possibility of false positives and false negative (fever as a symptom has not yet been developed although infected). We propose here another augmentation or an intermediate level of screening, depending on the usage, based on assessing olfactory functions. It is established with multiple publications reporting degrading (hyposmia) or loss of sense (anosmia) of smell for infected patients. This paper presents a recently developed technology and device that uses olfactory sense detection using a novel device that is capable of generating precise level of smell digitally, and repeatedly in contactless manner. The device is designed to work with a mobile app that can trigger selected smell or combinations and further can be programmed to give standard or customised smell tests. Estimated capital and running cost are low since one (out of 25) canister filled with of $0.1 \mathrm{ml}$ volume of essential smell oil can serve hundreds of tests. The prototype is tested to work for a desired smell generation and is available for further tweaking for rapid screening of subjects as per the requirements.
\end{abstract}

Keywords Covid smell test $\cdot$ Smell generator $\cdot$ Covid olfaction loss $\cdot$ Covid rapid pre-screening

\section{Introduction}

Evolution of recent pandemic of Coronavirus Disease (COVID-19) outbreak originated in China (Wu 2020) has a long lasting implications all over the world. Statistics (Situation reports 2020) show growth rate of infected patients over time and can be correlated to the measures taken by various countries. Massive testing and quarantine is one of the effective strategies emerging out of experience of countries such as South Korea (South Korea 2020). Testing methods currently employed can be classified into prescreening tests and screening actually for the presence of virus antigen (methods such as RT-PCR, antigen detection by MEMS or other sensors). Some of the screening methods are summarised along with their efficacy in (Liu et al. 2020). Although the later is the confirmatory test and the best way to be used ideally, it may or may not be feasible: based

Prasanna Gandhi

gandhi@iitb.ac.in

1 Indian Institute of Technology, Bombay, Powai, Mumbai 400076, India mainly on resources available and scale of the problem for countries such as India. The strategy could be to carry out pre-screening effectively and process fewer cases for actual screening for antigen detection. For example, pre-screening via temperature measurement as an indication of fever which was/is employed at the airport, entry to educational institutes such as IIT Bombay, and so on. Pre-screening would be more relevant while life is returning to normalcy.

Recent literature, both in the domain of formal publications (Lechien 2020; Brann 2020) and some major initiatives in research (Sound of breath 2020) indicate several possible ways of pre-screening which could be very useful for mass screening. Some major ones are mentioned below:

1. Body temperature: Its indication of development of symptoms in infected patients in the form of fever. This test is very simple, non-contact determination, does not need skilled manpower, cost effective, time efficient, and useful where the symptoms are indeed developed in form of fever. There are false positives of people having developed fever from other possible causes. There are false negatives where fever symptoms have not yet 
been developed. Thus it alone cannot be sufficient for pre-screening.

2. Sound of breath: (Sound of breath 2020) This test is based on physical breath sound or cough sound change that happens specifically for patients infected with COVID-19. This test is in development using machine learning techniques where a large data set is required for training.

3. Smell sense: Recent literature (Lechien 2020; Brann 2020; Dalton 2004; Giacomelli 2020) along with several online published findings report possibility of using smell loss either anosmia or hyposmia as a possible means of detection for mass scale pre-screeing. Although exact pathway for loss has not yet been established for COVID-19, the method seems to hold a lot of potential based on published literature (Brann 2020), early experiences of patients who tested positive, expert opinions (Hopkins 2020) and the fact that some other similar viruses are indeed found to have Postviral Olfactory Dysfunction (PVOD) (Suzuki 2007; Dalton 2004). These cases which are relatively low in numbers can form false positives for the smell sense way of prescreening. Anosmia reporting tool (COV 2020) is available online although data from it is not published yet.

\section{Smell Assessing Devices}

\section{Currently Available}

There are two commercially available smell tests/devices for smell:

1. Smell Identification Test ${ }^{\mathrm{TM}}$ (Smell identification test 2020): This test consist of four booklet, each contained 10 scratch card. The scratch area has micro encapsulated odorant. Test subject has to scratch the card gently to sniff the odour and has to identify the test item listed on that scratch card. Once Test subject has enter all 40 response, "The Smell Identification Test ${ }^{\mathrm{TM}}$ Administration" Manual is used to score the test. It gives indication of smell loss anosmia or mild, moderate, or severe hyposmia from manual.

2. Threshold Test with Sniff Stick (Sniffing sticks 2020): The olfactory threshold test determines the highest dilution of $\mathrm{N}$-butanol by test subject. The sniff stick consist of pens whose tips are soaked with $4 \mathrm{ml}$ of odorant fluid dissolved in propylene glycol at different concentration. The subjects responses are recorded on the grid form by varying concentration multiple times via an algorithm specified.

Both of these tests are non digital in nature and take long time for subjects to test and their purpose is different.

\section{Proposed Smell Testing Devices}

We propose two levels of the smell testing devices as mentioned below. They both can help pre-screening of infection before other symptoms are developed, in much faster way than tests available in the literature.

1. Simple smell scanning: This would consist of several small containers containing dry powder of substances such as cardamom, kewra, lemon, vanilla and pineapple and a subject is required to identify. The selection of scent and concentration is based on standard "University of Pennsylvania Smell Identification Test" and "Indian smell identification test" (George 2013; Jackman 2005). Also standards can be set using test on control groups to get minimum concentration required to be detected by viral infected people. Smell concentration can be controlled by single or multiple layers of filters put on the lid from where a subject wearing mask is made to smell and identify with an Android or iPhone based application and data be collected via Bluetooth at a central location. This test can be designed in a similar manner to the medically approved test proposed in (Smell identification test 2020) and (Sniffing sticks 2020) but can work much faster. This can be booth based test that can be self administered by subjects or can be given by unskilled or semiskilled person in several locations or even at home.

2. A novel automated digital smell generator: More sophisticated version of the test could be the digital scent or smell projector or generator developed recently by the group of authors as a part of a startup grant under NidhiPrayas scheme of DST. The device was originally developed for generating smell sense at demand or digitizing the smell experience for reproducing in various applications such as multi-sense real life experience for gaming, entertainment industry, experience in personal gadgets such as car and offices and so on. The current working prototype consists of the following subsystems (see Fig. 1 for schematic and picture of actually fabricated device):

1. Scent storage system or cartridge (The cartridge is use and throw type.)

2. Digital release on demand system which includes release elements and mixer

3. Power electronics of the driver circuit

4. Microcontroller to enable digital release

5. Mobile based app which will command the generator based on user need. This will be done via Bluetooth connection to microcontroller

6. Product package design 


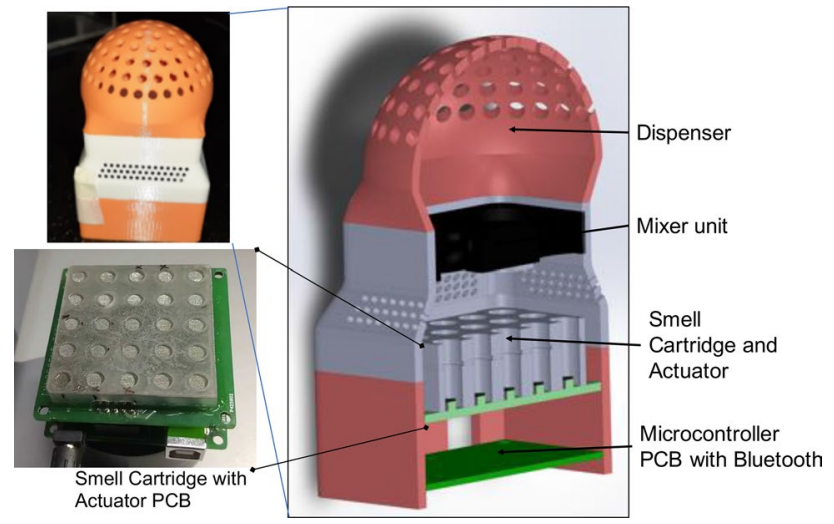

Fig. 1 Actually fabricated digital smell projector along with its schematic diagram

A cartridge (with $5 \times 5$ canisters) can hold 25 smell agents which can be dispensed individually or in accurate proportions all by a mobile app. A standard Indian smell test (Jackman 2005) or a customised smell test with concentrated oils of commonly known substances Lemon, Garlic, Rose, Orange, Sandalwood can be developed. As an example, 4 random combinations (two at each time) of smells of oils mentioned above can be digitally generated by mobile app for testing. Inability to correctly identify at least two combinations out of 4 may ascertain anosmia symptoms. Normally user will communicate via bluetooth or wifi protocol with generator with an app on his/her mobile and take the smell test. Response of user will be registered by the software and feedback will be provided to user as well as person administering the test. Thus, the test can be completely automated without need for skilled health worker. The dispenser part in this system needs replacement modification to suit smell test for COVID-19 considering safety of subjects. One such modification is detachable funnel through which subject can breath smell in. Once test is over funnel can be replaced with a new one. There is provision of a fan (operated digitally through software app) in mixer unit (see Fig. 1) to flush out all the smell molecules from the mixer area to purge the older scents between two tests. Expected purge time is around $15 \mathrm{~s}$. Depending on protocols established, we envisage overall results for the proposed test to be available within a few minutes. The device can be sanitised between two subjects by exposing the device surface to UV light as per protocol. Although it is not as rapid as the temperature screening it would be much it would provide an additional way for scrutiny enhancing reliability of identification of potential COVID-19 cases. One canister is currently designed to hold $0.1 \mathrm{ml}$ of oil which can be useful for 100s of tests. Estimated cost of smell gen- erator device is between Rs 3000-4000 per unit with medium scale of production. The replaceable cartridge (with 25 canisters) would be costing around Rs 200-300. Multiple devices can be deployed to bring down relative test time since the testing can be fully automated. Possible application areas could be airports, OPD clinics, and company premises to name a few.

In conclusion, this paper presents a method and device technology for rapid pre-screening tests for Covid-19. The proposed low cost method is based on recently reported loss of smell (Anosmia or hyposmia). The device which was developed for other application, can be readily modified for rapid pre-screening.

Acknowledgements Authors would like to acknowledge partial funding for this work from Nidhi Prayas Grant of DST and also support of Venture Center at NCL where the work was carried out.

\section{References}

Brann DH, Tsukahara T, Weinreb C, Logan DW, Datta SR (2020) Nonneural expression of SARS-CoV-2 entry genes in the olfactory epithelium suggests mechanisms underlying anosmia in COVID19 patients. bioRxiv. https://doi.org/10.1101/2020.03.25.009084

COV (2020) Covid-19 anosmia reporting tool. https://www.entne t.org/content/reporting-tool-patients-anosmia-related-covid-19. Accessed on 04 Aug 2020

Dalton P (2004) Olfaction and anosmia in rhinosinusitis. Curr Allergy Asthma Rep 4(3):230-236. https://doi.org/10.1007/s1188 2-004-0031-3

George J et al (2013) Use of Indian smell identification test for evaluating olfaction in idiopathic Parkinson's disease patients in India. Neurol India. https://doi.org/10.4103/0028-3886.117598

Giacomelli A et al (2020) Self-reported olfactory and taste disorders in SARS-CoV-2 patients: a cross-sectional study. Clin Infect Dis. https://doi.org/10.1093/cid/ciaa330

Hopkins C, Kumar N (2020) Loss of sense of smell as marker of covid-19 infection. https://www.entuk.org/loss-sense-smell-marke r-covid-19-infection-0. Accessed 21 Mar 2020

Jackman AH, Doty RL (2005) Utility of a three-item smell identification test in detecting olfactory dysfunction. Laryngoscope. https ://doi.org/10.1097/01.mlg.0000183194.17484.bb

Lechien JR et al (2020) Olfactory and gustatory dysfunctions as a clinical presentation of mild-to-moderate forms of the coronavirus disease (covid-19): a multicenter European study. Eur Arch Otorhinolaryngol. https://doi.org/10.1007/s00405-020-05965-1

Liu R, Fu A, Deng Z, Li Y, Liu T (2020) Promising methods for detection of novel coronavirus sars-cov-2. View 1(1):e4. https://doi. org/10.1002/viw2.4

Situation reports (2020) https://www.who.int/emergencies/diseases/ novel-coronavirus-2019/situation-reports. Accessed 23 Mar 2020 (Online)

Smell identification test (2020) (upsit)-smell products. https://senso nics.com/smell-identification-test-international-versions-avail able.html (Online)

Sniffing sticks for panel training on odour characters olfasense (2020) https://www.olfasense.com/odour-measurement-equipment/instr uments/sniffing-sticks-panel-training-characters (Online) 
Sound of breath (2020) https://healthcare-in-europe.com/en/news/ new-app-listens-to-the-sounds-of-covid-19.html. Accessed 07 Apr 2020 (Online)

South Korea measures (2020) https://www.theguardian.com/comme ntisfree/2020/mar/20/south-korea-rapid-intrusive-measures-covid -19. Accessed 20 Mar 2020 (Online)

Suzuki M et al (2007) Identification of viruses in patients with postviral olfactory dysfunction. Laryngoscope 117(2):272-277. https://doi. org/10.1097/01.mlg.0000249922.37381.1e
Wu Z, McGoogan JM (2020) Characteristics of and important lessons from the coronavirus disease 2019 (COVID-19) outbreak in china. JAMA 323(13):1239. https://doi.org/10.1001/jama.2020.2648

Publisher's Note Springer Nature remains neutral with regard to jurisdictional claims in published maps and institutional affiliations. 